On the persistence of lower-dimensional invariant hyperbolic tori for smooth Hamiltonian systems

This content has been downloaded from IOPscience. Please scroll down to see the full text. 2000 Nonlinearity 13189

(http://iopscience.iop.org/0951-7715/13/1/309)

View the table of contents for this issue, or go to the journal homepage for more

Download details:

IP Address: 124.16.154.203

This content was downloaded on 21/09/2015 at 01:41

Please note that terms and conditions apply. 


\title{
On the persistence of lower-dimensional invariant hyperbolic tori for smooth Hamiltonian systems
}

\author{
Debin Huang and Zengrong Liu \\ LNM, Institute of Mechanics, Academy of China, People's Republic of China \\ and \\ Mathematical Department, Shanghai University, Shanghai 201800, People's Republic of China $†$
}

Received 4 January 1999, in final form 29 September 1999

Recommended by V Baladi

\begin{abstract}
In this paper, sufficiently smooth Hamiltonian systems with perturbations are considered. By combining a smooth version of the Kolmogorov-Arnold-Moser theorem and the theory of normally hyperbolic invariant manifolds, we show that under the conditions of nonresonance and nondegeneracy, most hyperbolic invariant tori and their stable and unstable manifolds survive smoothly under sufficiently smooth autonomous perturbation. This result can be generalized directly to the case of time-dependent quasi-periodic perturbations. Finally, an example from geometrical optics is used to illustrate our method.
\end{abstract}

AMS classification scheme numbers: 58F27, 34C27, 58F30, 70H05

\section{Introduction}

It is well known that the Kolmogorov-Arnold-Moser (KAM) theorem is one of the mathematical breakthroughs of this century, and it has had a profound influence on physics and mechanics. Since the KAM theorem on KAM tori was established, there have been many generalizations similar to the KAM theorem, such as the generalizations to measure-preserving mappings $[2,18]$ and to quasi-periodic perturbation [8]. In particular, the generalization to lower-dimensional tori is still a subject of interest today. Firstly, Graff [6] considered the conservation of hyperbolic invariant tori, then elliptic lower-dimensional tori were investigated $[4,13]$ and, recently, further generalizations have emerged $[9,15]$.

We note the systems that these generalizations considered are all analytical Hamiltonian systems, and that they all used the basic strategy of an iterative KAM-type proof. However, for hyperbolic lower-dimensional tori, we want to weaken the analytical conditions to the finitely smooth case and sufficiently use the geometry of dynamical systems (i.e. invariant manifolds) to show similar results.

First, it is necessary to briefly recall Graff's work [6]. Consider $n$ degrees of freedom of a real-analytic Hamiltonian system near the origin

$$
\dot{z}=J D H(z)
$$

$\dagger$ Address for correspondence. 
where $z=\left(z_{1}, z_{2}, \ldots, z_{2 n}\right)$ and $J$ is the real canonical symplectic matrix

$$
j=\left(\begin{array}{cc}
0 & \mathrm{id} \\
-\mathrm{id} & 0
\end{array}\right)
$$

with 'id' an identity matrix, and

$$
H(z)=\sum_{i=2}^{\infty} H^{(i)}(z)
$$

where $H^{(i)}(z)$ is homogeneous of degree $i$. If $H^{(2)}(z)=\frac{1}{2}\langle z, A z\rangle,\langle\cdot, \cdot\rangle$ denotes the usual scalar product, then the linearized system near its fixed point, the origin, is

$$
\dot{z}=J A z \text {. }
$$

Suppose the eigenvalues of $J A\left( \pm \alpha_{1}, \pm \alpha_{2}, \ldots, \pm \alpha_{n}\right)$ are of the following form:

$$
\begin{array}{ll}
\operatorname{Re} \alpha_{s}=0 & 1 \leqslant s \leqslant k \\
\operatorname{Re} \alpha_{k+\lambda}>0 & 1 \leqslant \lambda \leqslant l .
\end{array}
$$

We introduce the notation $\alpha_{s}=\mathrm{i} \delta_{s}, 1 \leqslant s \leqslant k$, and assume $\delta_{s}(1 \leqslant s \leqslant k)$ are distinct and satisfy

$$
\sum_{s=1}^{k} j_{s} \delta_{s} \neq 0
$$

for all nonzero integer-valued vectors $j=\left(j_{1}, \ldots, j_{k}\right)$, with $1 \leqslant|j| \leqslant 4$, where $|j|=$ $\sum_{s=1}^{k}\left|j_{s}\right|$.

By some transformations, the Hamiltonian $H$ becomes

$$
\sum_{s=1}^{k} \delta_{s} y_{s}+\langle p, Q q\rangle+\frac{1}{2} \sum_{s, s^{\prime}=1}^{k} \tau_{s s^{\prime}} y_{s} y_{s^{\prime}}+K(u, v, p, q)
$$

where $Q$ is an $l \times l$ matrix satisfying the positivity condition

$$
\operatorname{Re}\langle\gamma, Q \gamma\rangle \geqslant \mu|\gamma|^{2}
$$

for all nonzero complex vectors $\gamma$ and some $\mu>0,2 y_{s}=u_{s}^{2}+v_{s}^{2}, \tau=\left(\tau_{s s^{\prime}}\right)$ is a real $k \times k$ matrix and $K$ is a power series. In (1.4), $K$ may be considered as a perturbation of the Hamiltonian

$$
\sum_{s=1}^{k} \delta_{s} y_{s}+\langle p, Q q\rangle+\frac{1}{2} \sum_{s, s^{\prime}=1}^{k} \tau_{s s^{\prime}} y_{s} y_{s^{\prime}} .
$$

Obviously, the Hamiltonian system afforded by (1.5) admits a family of $k$-dimensional invariant tori

$$
\begin{array}{lc}
\dot{x}_{5}=\omega_{s} \quad y_{s}=\tilde{y}_{s} & 1 \leqslant s \leqslant k \quad p=q=0 \\
\omega_{s}(\tilde{y})=\delta_{s}+\sum_{s^{\prime}=1}^{k} \tau_{s s^{\prime}} \tilde{y}_{s^{\prime}} & \tilde{y}=\left(\tilde{y}_{1}, \ldots, \tilde{y}_{k}\right) \text { is a vector of constants }
\end{array}
$$

and these tori admit a $(k+l)$-dimensional real-analytic Lagrangian stable manifold $M_{-}$and a Lagrangian unstable manifold $M_{+}$, consisting, respectively, of orbits approaching the torus tangentially at an exponential rate as $t \rightarrow+\infty$ and $t \rightarrow-\infty$.

In [6], Graff showed the following result by a Newton iteration procedure. 
Theorem 1.1. If in (1.4), det $\tau \neq 0$, then the invariant tori in (1.6) which satisfy the nondegeneracy condition

$$
|\langle j, \omega\rangle| \geqslant c|j|^{-N} \quad j=\left(j_{1}, \ldots, j_{k}\right) \quad \omega=\left(\omega_{1}, \ldots, \omega_{k}\right) \quad c>0 \quad N>k
$$

for all nonzero integer-valued vectors $j$, and their stable and unstable manifolds persist real analytically under perturbation, i.e. in (1.4).

Although the above result shows the analytic persistence of hyperbolic tori for analytic Hamiltonian systems, in some applications the Hamiltonian is only smooth, not analytic. So we think it is interesting to consider smooth Hamiltonian systems. In this paper, from the geometric viewpoint of dynamical systems, we show the smooth persistence of hyperbolic tori and their stable and unstable manifolds for sufficiently smooth Hamiltonian systems with the above conditions of nonresonance and nondegeneracy. Our method is to combine a smooth version of the KAM theorem with the theory of normally hyperbolic invariant manifolds [5] (see also [17]), instead of the rapidly convergent Newton iteration technique. Although this idea was referred to in [6], there it required the analytical perturbations due to not using the normally hyperbolic theory. Also, this result can be generalized directly to the case of timedependent quasi-periodic perturbations and the method may be used to show the persistence of lower-dimensional tori in a priori stable Hamiltonian systems [15] (the latter is very obvious, and will not concern us here). Finally, we use a simple example from geometrical optics to illustrate our method.

\section{Some elementary reviews}

First, we give a brief description of the invariant manifold in [5].

Consider a $C^{r}, r \geqslant 1$ vector field on $R^{n}$,

$$
\dot{x}=f(x) \quad x \in R^{n}
$$

with its flow denoted by $\phi_{t}(x)$. Suppose that (2.1) has an overflowing invariant manifold, $\bar{M}=M \cup \partial M$. By the term 'overflowing invariant' we mean that the vector field is tangential to $M$ and points strictly outward on $\partial M$, therefore all trajectories starting on $\partial M$ leave $\bar{M}$. to $\bar{M}$ :

Suppose we have the following continuous splitting of the tangent bundle of $R^{n}$ restricted

$$
\left.T R^{n}\right|_{\bar{M}}=T \bar{M} \oplus N^{s} \oplus N^{u}
$$

with the associated projections

$$
\begin{aligned}
& \Pi^{\mathrm{T}}:\left.T R^{n}\right|_{\bar{M}} \rightarrow T \bar{M} \\
& \Pi^{u}:\left.T R^{n}\right|_{\bar{M}} \rightarrow N^{u} \\
& \Pi^{s}:\left.T R^{n}\right|_{\bar{M}} \rightarrow N^{s} .
\end{aligned}
$$

We assume that the subbundles $T \bar{M} \oplus N^{u}$ and $T \bar{M} \oplus N^{s}$ are each invariant under the linearized flow $D \phi_{t}$ for all $t<0$. We also assume that for each $p \in \bar{M}, N_{p}^{u}$ is $u$ dimensional and $N_{p}^{s}$ is $s$ dimensional; therefore $\bar{M}$ is $n-(s+u)$ dimensional. 
Growth rates of vectors in these subbundles under the linearized dynamics are characterized by generalized Lyapunov-type numbers defined as follows:

$$
\begin{aligned}
& \lambda^{u}(p)=\limsup _{t \rightarrow-\infty}\left\|\Pi^{u} D \phi_{t}(p) \mid N_{p}^{u}\right\|^{-1 / t} \\
& v^{s}(p)=\limsup _{t \rightarrow-\infty}\left\|\Pi^{s} D \phi_{-t}\left(\phi_{t}(p)\right) \mid N_{p}^{s}\right\|^{-1 / t} \\
& \sigma^{s}(p)=\limsup _{t \rightarrow-\infty} \frac{\log \left\|\left.D \phi_{t}\right|_{M}(p)\right\|}{-\log \left\|\left.\Pi^{s} D \phi_{-t}\left(\phi_{t}(p)\right)\right|_{N_{p}^{s}}\right\|}
\end{aligned}
$$

where $\|\cdot\|$ is some matrix norm. The manifold $\bar{M}$ is called normally hyperbolic if for any point $p \in \bar{M}, \lambda^{u}(p), v^{s}(p)<1$ and $\sigma^{s}(p)<1 / r$ hold. (Note that this definition is also suitable for any invariant manifold.) For normally hyperbolic invariant manifolds and their unstable manifolds, we have the following persistence theorem in [5].

Theorem 2.1. Suppose $\bar{M}=M \cup \partial M$ is a $C^{r}$ normally hyperbolic manifold with boundary overflowing invariant under the vector field (2.1), and with $T \bar{M} \oplus N^{u}$ and $T \bar{M} \oplus N^{s}$ negatively invariant under the linearized flow $D \phi_{t}$, then there exists a $C^{r}$ manifold $W^{u}$ overflowing invariant under (2.1) such that $W^{u}$ contains $\bar{M}$ and is tangential to $T \bar{M} \oplus N^{u}$ along $\bar{M}$ with trajectories in $W^{u}$ approaching $\bar{M}$ as $t \rightarrow-\infty$. Moreover, $W^{u}$ is persistent underperturbation in the sense that for any $C^{r}$ vector field $f_{\varepsilon}(x) \mathrm{O}(\varepsilon) C^{1}$-close to $f(x)$ with $\varepsilon$ sufficiently small, there is a manifold $W_{\varepsilon}^{u}$ overflowing invariant under $f_{\varepsilon}(x)$ which is $C^{r}$ diffeomorphic to $W^{u}$ and has the same dimension as $W^{u}$.

Further, we give the two additional type numbers:

$$
\begin{aligned}
& \sigma^{c u}(p)=\limsup _{t \rightarrow-\infty}\left\|\left.D \phi_{-t}\right|_{M}\left(\phi_{t}(p)\right)\right\|^{-1 / t}\left\|\left.\Pi^{u} D \phi_{t}(p)\right|_{N_{p}^{u}}\right\|^{-1 / t} \\
& \sigma^{s u}(p)=\limsup _{t \rightarrow-\infty}\left\|\left.\Pi^{u} D \phi_{t}(p)\right|_{N_{p}^{u}}\right\|^{-1 / t}\left\|\left.\Pi^{s} D \phi_{-t}\left(\phi_{t}(p)\right)\right|_{N_{p}^{s}}\right\|^{-1 / t} .
\end{aligned}
$$

Then we have the following unstable manifold foliation theorem, see [17].

Theorem 2.2. Let $\bar{M}$ be as in theorem 2.1. Moreover, suppose $\sigma^{c u}(p)<1$ and $\sigma^{\text {su }}(p)<1$ for every $p \in \bar{M}$. Then there exists an $n-(s+u)$-parameter family $F^{u} \equiv \cup_{p \in M} f^{u}(p)$ of $u$-dimensional surfaces $f^{u}(p)$, such that

(1) $F^{u}$ is a negatively invariant family.

(2) $f^{u}(p)$ are $C^{r}$.

(3) $f^{u}(p)$ are tangent at $p$ to the embedding of $N_{p}^{u}$ into $R^{n}$.

(4) There exist $C_{1}, C_{2}>0$ such that if $q \in f^{u}(p)$ then

$$
\left\|\phi_{-t}(q)-\phi_{-t}(p)\right\|<C_{1} \mathrm{e}^{C_{2} t} \quad \text { for any } t \geqslant 0 .
$$

(5) Suppose $q \in f^{u}(p)$ and $q^{\prime} \in f^{u}\left(p^{\prime}\right), p \neq p^{\prime}$, then

$$
\frac{\left\|\phi_{-t}(q)-\phi_{-t}(p)\right\|}{\left\|\phi_{-t}\left(q^{\prime}\right)-\phi_{-t}(p)\right\|} \rightarrow 0 \quad \text { as } \quad t \rightarrow \infty
$$

and $f^{u}(p)$ and $f^{u}\left(p^{\prime}\right)$ do not intersect.

(6) $f^{u}(p)$ are $C^{r}$ with respect to the basepoint $p$.

(7) $F^{u}=W_{\mathrm{loc}}^{u}(M)$ which is a local unstable manifold of $M$.

(8) These fibres $f^{u}(p)$ persist and remain $C^{r}$ under $C^{r}$ perturbations. 
Remark 2.1. Statement (4) of theorem 2.2 implies that we can identify the unstable manifolds of objects in $M$ from the unstable manifold of $M$; and the above results can be applied to inflowing invariant manifolds. In this case, the generalized Lyapunov-type numbers are computed using the time-reversed flow with the limits taken as $t \rightarrow+\infty$ and the 'overflowing invariant' is replaced by the 'inflowing invariant'. Also $N^{u}$ and $W^{u}$ are replaced by $N^{s}$ and $W^{s}$, with $N^{s}$ taken to be a positively invariant subbundle under $D \phi_{t}$. Then the above theorem can be recast as the stable manifold theorem.

At the end of this section, we give the spirit of the KAM theorem briefly.

Theorem 2.3 (Classical KAM theorem [1]). Consider a near-integrable Hamiltonian system with the Hamiltonian

$$
H(I, \varphi)=N_{0}(I)+P_{0}(I, \varphi)
$$

where $I=\left(I_{1}, I_{2}, \ldots, I_{n}\right)$ are the action variables, $\varphi=\left(\varphi_{1}, \varphi_{2}, \ldots, \varphi_{n}\right)$ are the angle variables and $P_{0}$ is sufficiently small and is referred to as a small perturbation. Suppose

(i) $H(I, \varphi)$ is analytic,

(ii) $N_{0}(I)$ satisfies the condition of nondegeneracy

$$
\operatorname{det}\left(\frac{\partial^{2} N_{0}}{\partial I^{2}}\right) \neq 0
$$

then the invariant tori of the unperturbed Hamiltonian $N_{0}(I)$, with

$$
\left|\left\langle\frac{\partial N_{0}}{\partial I}(I), j\right\rangle\right| \geqslant c|j|^{-N}
$$

for all nonzero integer-valued vectors $j$, persist in (2.3).

Remark 2.2. The analogous theorem for vector fields with finitely many derivatives was first given by Moser [14]. Moser's result can be adapted to $C^{r}$ Hamiltonian vector fields generated by the form of (2.3) with $r \geqslant 2 n-1$, see [12].

\section{Main results and proof}

On the basis of the introduction in section 1, we consider directly the following $C^{r}$ Hamiltonian vector field:

$$
\begin{aligned}
\dot{x} & =\frac{\partial H_{0}}{\partial y}(x, y, I)+\varepsilon \frac{\partial H_{1}}{\partial y}(x, y, I, \varphi, \varepsilon) \\
\dot{y} & =-\frac{\partial H_{0}}{\partial x}(x, y, I)-\varepsilon \frac{\partial H_{1}}{\partial x}(x, y, I, \varphi, \varepsilon) \\
\dot{I} & =-\varepsilon \frac{\partial H_{1}}{\partial \varphi}(x, y, I, \varphi, \varepsilon) \\
\dot{\varphi} & =\frac{\partial H_{0}}{\partial I}(x, y, I)+\varepsilon \frac{\partial H_{1}}{\partial I}(x, y, I, \varphi, \varepsilon)
\end{aligned}
$$

where $x=\left(x_{1}, x_{2}, \ldots, x_{l}\right) \in R^{l}, y=\left(y_{1}, y_{2}, \ldots, y_{l}\right) \in R^{l}, I=\left(I_{1}, I_{2}, \ldots, I_{k}\right) \in U$ which is an open set in $R^{k}, \varphi=\left(\varphi_{1}, \varphi_{2}, \ldots, \varphi_{k}\right) \in T^{k}$ and $\varepsilon$ is sufficiently small. Our assumptions are

$$
\frac{\partial H_{0}}{\partial y}(0,0, I)=\frac{\partial H_{0}}{\partial x}(0,0, I)=0
$$


(A3) the eigenvalues of

$$
\operatorname{det}\left[\frac{\partial^{2} H_{0}}{\partial I^{2}}(0,0, I)\right] \neq 0
$$

$$
J \frac{\partial^{2} H_{0}}{\partial X^{2}}(0,0, I), \quad X=(x, y)
$$

have nonzero real parts.

Now consider the unperturbed system of (3.1)

$$
\begin{aligned}
\dot{x} & =\frac{\partial H_{0}}{\partial y}(x, y, I) \\
\dot{y} & =-\frac{\partial H_{0}}{\partial x}(x, y, I) \\
\dot{I} & =0 \\
\dot{\varphi} & =\frac{\partial H_{0}}{\partial I}(x, y, I) .
\end{aligned}
$$

From (A1), in system (3.2) there exists a $2 k$-dimensional invariant manifold denoted by

$$
M=\left\{(x, y, I, \varphi) \mid x=y=0, I \in U, \varphi \in T^{k}\right\} .
$$

The vector field (3.2) restricted to $M$ is

$$
\dot{I}=0 \quad \dot{\varphi}=\frac{\partial H_{0}}{\partial I}(0,0, I) \equiv \omega(I)
$$

where $\omega(I)=\left(\omega_{1}(I), \omega_{2}(I), \ldots, \omega_{k}(I)\right)$. From (3.4), $M$ actually consists of a $k$-parameter family of $k$-dimensional tori; and according to (A3), $M$ has $C^{r}(2 k+l)$-dimensional stable and unstable manifolds denoted by $W^{s}(M)$ and $W^{u}(M)$, respectively. Moreover, by theorem 2.2 and the generalized-type numbers obtained below, the stable and unstable manifolds of some $k$-dimensional tori in (3.4) may be identified from $W^{s}(M)$ and $W^{u}(M)$, respectively.

Next, we show that $M$ is normally hyperbolic. The linearized vector field of (3.2) along $M$ is given by

$$
\left(\begin{array}{c}
\delta \dot{X} \\
\delta \dot{I} \\
\delta \dot{\varphi}
\end{array}\right)=\left(\begin{array}{ccc}
J \frac{\partial^{2} H_{0}}{\partial X^{2}}(0,0, I) & 0 & 0 \\
0 & 0 & 0 \\
\frac{\partial^{2} H_{0}}{\partial I \partial X}(0,0, I) & \frac{\partial^{2} H_{0}}{\partial I^{2}}(0,0, I) & 0
\end{array}\right)\left(\begin{array}{c}
\delta X \\
\delta I \\
\delta \varphi
\end{array}\right)
$$

where $X=(x, y)$ and $\delta X, \delta I$ and $\delta \varphi$ represent variations about orbits on $M$. From (3.5) we obtain the linearized flow about $M, D \phi_{t}$. This is given by

$D \phi_{t}(0,0, I, \varphi)=$

$$
\left(\begin{array}{ccc}
\exp \left[J \frac{\partial^{2} H_{0}}{\partial X^{2}}(0,0, I) t\right] & 0 & 0 \\
0 & \operatorname{id}_{k} & 0 \\
s \frac{\partial^{2} H_{0}}{\partial I \partial X}(0,0, I)\left[J \frac{\partial^{2} H_{0}}{\partial X^{2}}(0,0, I)\right]^{-1} \exp \left[J \frac{\partial^{2} H_{0}}{\partial X^{2}}(0,0, I) t\right] & \frac{\partial^{2} H_{0}}{\partial I^{2}}(0,0, I) t & \operatorname{id}_{k}
\end{array}\right)
$$

where $i_{k}$ denotes the $k \times k$ identity matrix. 
Note that vectors tangential to $M$ have zero $X$ component, so the second and third columns of (3.6) span $T_{p} M$ for any $p \in M$. Thus the projection onto $T_{p} M$ is given by

$$
D \phi_{t}(p) \Pi^{\mathrm{T}}=\left(\begin{array}{ccc}
0 & 0 & 0 \\
0 & \mathrm{id}_{k} & 0 \\
0 & \frac{\partial^{2} H_{0}}{\partial I^{2}}(0,0, I) t & \mathrm{id}_{k}
\end{array}\right) .
$$

Now, we want to decompose $\left.T R^{2 k+2 l}\right|_{M}$ into three subbundles. First, consider the equation of the $X$ component in (3.5) regarding $I$ as fixed

$$
\delta \dot{X}=J \frac{\partial^{2} H_{0}}{\partial X^{2}}(0,0, I) \delta X \quad I \in U .
$$

By (A3), for each $I \in U, R^{2 l}$ splits into two $l$-dimensional subspaces $E^{s}(I)$ and $E^{u}(I)$, corresponding to the stable and unstable subspaces of (3.8). Consider the following two disjoint unions:

$$
\begin{aligned}
& N^{s}=\bigcup_{I \in U}\left(E^{s}(I), 0\right) \\
& N^{u}=\bigcup_{I \in U}\left(E^{u}(I), 0\right)
\end{aligned}
$$

where ' 0 ' denotes a zero vector in $R^{2 k}$. Then we have

$$
\left.T R^{2 l+2 k}\right|_{M}=T M \oplus N^{s} \oplus N^{u} .
$$

Obviously $T M \oplus N^{s}$ and $T M \oplus N^{u}$ are two invariant subbundles under $D \phi_{t}$, i.e. (3.6).

We now compute the generalized Lyapunov-type numbers associated in the context of section 2. For any $p \in M$, using (3.7) and (3.8), we obtain

$$
\begin{array}{ll}
\lambda^{u}(p)=\mathrm{e}^{-\lambda_{u}(I)} & v^{s}(p)=\mathrm{e}^{\lambda_{s}(I)} \quad \sigma^{s}(p)=0 \\
\sigma^{c u}(p)=\mathrm{e}^{-\lambda_{u}(I)} & \sigma^{s u}(p)=\mathrm{e}^{-\lambda_{u}(I)+\lambda_{s}(I)}
\end{array}
$$

where $\lambda_{u}(I)$ is the smallest real part of any of the $l$ eigenvalues of $J \partial^{2} H_{0}(0,0, I) / \partial X^{2}$ which have positive real parts, and $\lambda_{s}(I)$ is the largest real part of any of the $l$ eigenvalues of $J \partial^{2} H_{0}(0,0, I) / \partial X^{2}$ which have negative real parts. Recalling (A3), we have

$$
-\lambda_{u}(I), \lambda_{s}(I)<0 \quad \forall I \in U
$$

and therefore

$$
\begin{array}{lll}
\lambda(p)<1 & v(p)<1 & \sigma(p)=0<\frac{1}{r} \\
\sigma^{c u}(p)<1 & \sigma^{s u}(p)<1 & \forall p \in \bar{M} .
\end{array}
$$

The same conclusion holds for $T M \oplus N^{s}$ under the time-reversed vector field from remark 2.1. So the normally hyperbolic properties of $M$ are proved, and $M$ satisfies the conditions in theorem 2.2.

Now we show the persistence of $M, W^{s}(M)$ and $W^{u}(M)$ by using theorem 2.1. However, since $M$ is neither overflowing nor inflowing invariant due to the fact that vector field (3.2) is identically zero on $\partial M$, there is a slight difficulty. By some technique, we obtain the following result. 
Proposition 3.1. There exists $\varepsilon_{0}>0$, such that for $0<\varepsilon<\varepsilon_{0}$, system (3.1) possesses a $C^{r}$ $2 k$-dimensional invariant manifold

$$
M_{\varepsilon}=\left\{(x, y, I, \varphi) \mid x=\mathrm{O}(\varepsilon), y=\mathrm{O}(\varepsilon), I \in \tilde{U} \subset U, \varphi \in T^{k}\right\}
$$

which is $C^{r}$ smooth in $I, \varphi, \varepsilon$, where $\tilde{U} \subset U$ is a compact $k$-dimensional set including the origin ' $O$ '. Moreover, $M_{\varepsilon}$ has $C^{r}$ stable and unstable manifolds, $W^{s}\left(M_{\varepsilon}\right)$ and $W^{u}\left(M_{\varepsilon}\right)$, which are $C^{r}$ diffeomorphic to $W^{s}(M)$ and $W^{u}(M)$ locally, respectively.

Proof. Let $\tilde{U} \subset U$ be a compact set including the origin ' 0 '. Choose open convex sets $U_{0}$ and $U_{1}$ such that $\tilde{U} \subset U_{0} \subset U_{1} \subset U$ with closure $\left(U_{0}\right) \subset U_{1}$. Construct a $C^{\infty}$ 'bump' function [16]

$$
g: R^{k} \rightarrow R
$$

such that

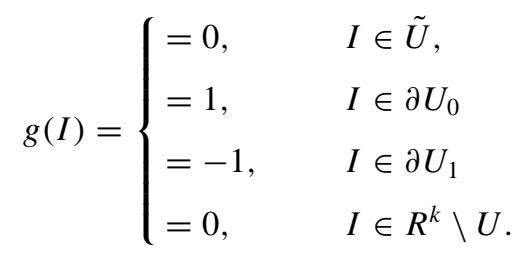

Now consider the modfied vector field of (3.2)

$$
\begin{aligned}
\dot{x} & =\frac{\partial H_{0}}{\partial y}(x, y, I) \\
\dot{y} & =-\frac{\partial H_{0}}{\partial x}(x, y, I) \\
\dot{I} & =\tilde{\varepsilon} g(I) I \\
\dot{\varphi} & =\frac{\partial H_{0}}{\partial I}(x, y, I)
\end{aligned}
$$

for some $\tilde{\varepsilon}>0$. Let $\tilde{M}, M_{0}$ and $M_{1}$ be subsets of $M$ for which $I$ is restricted to lie in $\tilde{U}$, $U_{0}$ and $U_{1}$, respectively. Then $\tilde{M} \subset M_{0} \subset M_{1} \subset M$, and from the above discussions the following statements are obvious:

(i) $M_{0}$ is an overflowing manifold under (3.14) satisfying the conditions of theorem 2.1.

(ii) $M_{1}$ is an inflowing manifold under (3.14) satisfying the conditions of theorem 2.1 under the time-reversed vector field.

In addition, from (3.13), equations (3.14) and (3.12) are identical for $I \in \tilde{U}$, so it follows from theorem 2.1 that the perturbed system (3.1) possess a $C^{r}$-invariant manifold $M_{\varepsilon}$ which is $\mathrm{O}(\varepsilon) C^{r}$ close to $M$. Moreover, there exist invariant manifolds $W^{s}\left(M_{\varepsilon}\right)$ and $W^{u}\left(M_{\varepsilon}\right)$ which are $C^{r}$ close to $W^{s}(M)$ and $W^{u}(M)$ locally, respectively. The proposition is proved.

Remark 3.1. Actually, from the above proof, the invariant property of $M_{\varepsilon}$ is slightly different from the usual definition of the invariant, since points starting from $M_{\varepsilon}$ may leave $M_{\varepsilon}$ by crossing its boundary. But this will cause us little concern because we consider only the invariant tori in $M_{\varepsilon}$, not the whole $M_{\varepsilon}$.

Next, we investigate the persistence of the invariant tori under the perturbation. In order to explore the dynamics on $M_{\varepsilon}$, we first prove a technical lemma which will be stated in its full generality. Throughout the lemma, $\mathrm{d}$ will refer to exterior differentiation and $i_{F} \varpi$ will denote the interior product of the vector field $F$ with the form $\varpi$. 
Lemma 3.1. Let $(P, \varpi)$ be a $(2 k+2 l)$-dimensional symplectic manifold with local coordinates $X=\left(x_{1}, x_{2}, \ldots, x_{l}, y_{1}, y_{2}, \ldots, y_{l}\right), Z=\left(z_{1}, z_{2}, \ldots, z_{k}, \varphi_{1}, \varphi_{2}, \ldots, \varphi_{k}\right)$, with the symplectic form $\varpi=\sum_{i=1}^{l} \mathrm{~d} y_{i} \wedge \mathrm{d} x_{i}+\sum_{j=1}^{k} \mathrm{~d} z_{j} \wedge \mathrm{d} \varphi_{j}$. Consider a one-parameter family of $2 k$ dimensional $C^{s}$ submanifolds $P_{\varepsilon} \subset P$, with $1 \leqslant s \leqslant r$ and $\varepsilon \in\left[0, \varepsilon_{0}\right]$, of the form

$$
P_{\varepsilon}=\{(X, Z) \in P \mid X=h(z)+\varepsilon b(Z, \varepsilon)\}
$$

where $z \equiv\left(z_{1}, z_{2}, \ldots, z_{k}\right), h=\left(h_{1}, h_{2}, \ldots, h_{2 l}\right)$ and $b \equiv\left(b_{1}, b_{2}, \ldots, b_{2 l}\right)$ are (locally defined) $C^{s+1}$ functions with $h_{i}: R^{k} \rightarrow R, b_{i}: R^{2 k} \rightarrow R, i=1, \ldots, 2 l$. Further, assume that $H$ is a $C^{r}$ function on $P$, and for any $\varepsilon \in\left[0, \varepsilon_{0}\right], P_{\varepsilon}$ is an integral manifold for the Hamiltonian vector field $F_{H}: P \rightarrow T P$ defined by

$$
i_{F_{H}} \varpi=\mathrm{d} H \text {. }
$$

Then for $\varepsilon$ sufficiently small,

(i) $\left(P_{\varepsilon}, \varpi_{\varepsilon}\right)$ is a $2 k$-dimensional symplectic $C^{s}$ manifold with $\varpi_{\varepsilon}=\left.\varpi\right|_{P_{\varepsilon}}$,

(ii) $F_{\varepsilon}=\left.F_{H}\right|_{P_{\varepsilon}}$ is a Hamiltonian vector field on $P_{\varepsilon}$ with Hamiltonian $H_{\varepsilon}=\left.H\right|_{P_{\varepsilon}}$, i.e.

$$
i_{F_{\varepsilon}} \varpi_{\varepsilon}=\mathrm{d} H_{\varepsilon} .
$$

Proof. We first show that $\varpi_{\varepsilon}$ defines a symplectic structure on $P_{\varepsilon}$. On $P_{\varepsilon}$, we have

$$
\begin{array}{lll}
\mathrm{d} x_{i}=\varepsilon D_{\varphi} b_{i} \mathrm{~d} \varphi+D_{z}\left(h_{i}+\varepsilon b_{i}\right) \mathrm{d} z & \varphi=\left(\varphi_{1}, \varphi_{2}, \ldots, \varphi_{k}\right) \quad i=1, \ldots, l \\
\mathrm{~d} y_{j}=\varepsilon D_{\varphi} b_{j+l} \mathrm{~d} \varphi+D_{z}\left(h_{j+l}+\varepsilon b_{j+l}\right) \mathrm{d} z & j=1, \ldots, l .
\end{array}
$$

By an easy calculation, we have

$$
\varpi_{\varepsilon}=(1+\mathrm{O}(\varepsilon)) \sum_{i=1}^{k} \mathrm{~d} z_{i} \wedge \mathrm{d} \varphi_{i}
$$

which implies that, for $\varepsilon$ sufficiently small, $\varpi_{\varepsilon}$ is a nondegenerate 2-form on $P_{\varepsilon}$. Let $e_{\varepsilon}=\left(h+\varepsilon b, \mathrm{id}_{2 k}\right): R^{2 k} \rightarrow P$ be the embedding of $P_{\varepsilon}$, where $\mathrm{id}_{2 k}$ denotes the $2 k$-dimensional identity map. Then

$$
\mathrm{d} \varpi_{\varepsilon}=\mathrm{d}\left(e_{\varepsilon}^{*} \varpi\right)=e_{\varepsilon}^{*} \mathrm{~d} \varpi=0
$$

which shows that $\varpi_{\varepsilon}$ is closed on $P_{\varepsilon}$ (where $e_{\varepsilon}^{*}$ denotes the pullback of $e_{\varepsilon}$, see [11]). Equations (3.16) and (3.17) together imply (i) of the lemma.

To show (ii), consider any $p_{\varepsilon} \in e_{\varepsilon}^{-1}\left(P_{\varepsilon}\right)$ and $u \in T_{P_{\varepsilon}} R^{2 k}$. In the context of the differential form we have

$$
\begin{aligned}
i_{F_{\varepsilon}} \varpi_{\varepsilon}\left[p_{\varepsilon}\right](u) & =e_{\varepsilon}^{*} \varpi\left[p_{\varepsilon}\right]\left(e_{\varepsilon}^{*} F_{H}\left(p_{\varepsilon}\right), u\right) \\
& \left.=\varpi\left[e_{\varepsilon}\left(p_{\varepsilon}\right)\right] \mathrm{d} e_{\varepsilon} \mathrm{d} e_{\varepsilon}^{-1} F_{H}\left(e_{\varepsilon}\left(p_{\varepsilon}\right)\right), \mathrm{d} e_{\varepsilon} u\right) \\
& \left.=\varpi\left[e_{\varepsilon}\left(p_{\varepsilon}\right)\right]\left(F_{H}\left(e_{\varepsilon}\left(p_{\varepsilon}\right)\right), \mathrm{d} e_{\varepsilon} u\right)=\mathrm{d} H\left[e_{\varepsilon}\left(p_{\varepsilon}\right)\right] \mathrm{d} e_{\varepsilon} u\right) \\
& =\mathrm{d}\left(e_{\varepsilon}^{*} H\right)\left[p_{\varepsilon}\right](u)=\mathrm{d} H_{\varepsilon}\left[p_{\varepsilon}\right](u)
\end{aligned}
$$

which concludes the proof.

Remark 3.2. The above lemma is easy but not trivial because it is not obviously true for all invariant manifolds of the Hamiltonian dynamics, e.g. for stable and unstable manifolds of equilibrium. This is easily seen from the structure of the stable and unstable subspaces of the equilibrium in the linearized problem [10]. 
We now apply the above lemma to $M_{\varepsilon}$ given in proposition 3.1 and obtain proposition 3.2.

Proposition 3.2. Consider system (3.1) restricted to its invariant manifold $M_{\varepsilon}$. For $\varepsilon$ sufficiently small, the restricted dynamics to $M_{\varepsilon}$ is Hamiltonian with the Hamiltonian

$$
H_{\varepsilon}=H_{0}(0,0, I)+\varepsilon H_{1}(0,0, I, \varphi, 0)+\mathrm{O}\left(\varepsilon^{2}\right)
$$

and with the restricted symplectic form

$$
\varpi_{\varepsilon}=(1+\mathrm{O}(\varepsilon)) \sum_{i=1}^{k} \mathrm{~d} I_{i} \wedge \mathrm{d} \varphi_{i} .
$$

Proof. This follows directly from lemma 3.1, proposition 3.1 and (A1).

Therefore, from proposition 3.2 , system (3.1) restricted to $M_{\varepsilon}$ is a $2 k$-dimensional Hamiltonian system

$$
\begin{aligned}
\dot{I} & =-\varepsilon \frac{\partial \tilde{H}_{1}}{\partial \varphi}(I, \varphi)+\mathrm{O}\left(\varepsilon^{2}\right) \\
\dot{\varphi} & =\omega(I)+\varepsilon \frac{\partial \tilde{H}_{1}}{\partial I}(I, \varphi)+\mathrm{O}\left(\varepsilon^{2}\right)
\end{aligned}
$$

where $\tilde{H}_{1}(I, \varphi)=H_{1}(0,0, I, \varphi, 0), \omega(I)$ is given in (3.4). Thus if the assumption (A2) holds, we may consider the persistence of the invariant tori in (3.19) by applying the KAM theorem. However, equation (3.19) is not analytic, so we must investigate the order of differentiability of (3.19) in order to use the conclusion of remark 2.2. From proposition 3.1, $M_{\varepsilon}$ is a $C^{r}$ manifold, but from lemma 3.1, the orders of differentiability of the functions $h$ and $b$ must be larger than the order of manifold $M_{\varepsilon}$, so $\left(M_{\varepsilon}, \varpi_{\varepsilon}\right)$ is a $C^{r-1}$ symplectic manifold at most. Therefore, to be able to use the smooth version of the KAM theorem for (3.19), we need the following assumption:

$$
r-1>2 k-1 \quad \text { i.e. } r>2 k .
$$

Finally, from the smooth version of the KAM theorem, proposition 3.1 and theorem 2.2, we obtain the main result.

Theorem 3.1. For the $C^{r}$ Hamiltonian vector field (3.1), suppose (A1)-(A4) hold, then for $\varepsilon$ small enough, the $k$-parameter family of $k$-dimensional invariant tori in (3.2)

$$
\begin{aligned}
& x=y=0 \\
& I=I_{0} \\
& \varphi=\omega\left(I_{0}\right) t+\varphi_{0}
\end{aligned}
$$

with

$$
\left|\left\langle\omega\left(I_{0}\right), j\right\rangle\right| \geqslant c|j|^{-N}
$$

for all nonzero integer-valued $k$-vectors $j$ where $c$ is a positive constant and $N>k-1$, and their stable and unstable manifolds persist in (3.1). Moreover, the continuations of the persistence are differentiable.

Proof. This follows directly from the preceding results. 


\section{Time-dependent quasi-periodic perturbation}

In the past, most work was focused on the Hamiltonian systems with autonomous perturbation. However, recently, the analytic Hamiltonian systems with time-dependent quasi-periodic perturbations whose Hamiltonian is of the form

$$
H(I, \varphi)=H_{0}(I)+\varepsilon H_{1}(I, \varphi, \tilde{\varphi}, \varepsilon)
$$

where $\tilde{\varphi}=\left(\tilde{\varphi}_{1}, \tilde{\varphi}_{2}, \ldots, \tilde{\varphi}_{s}\right)$ and $\tilde{\varphi}$ is quasi-periodic with respect to the time with the frequency $\tilde{\omega}=\left(\tilde{\omega}_{1}, \tilde{\omega}_{2}, \ldots, \tilde{\omega}_{s}\right)$, have been extensively studied because these kinds of Hamiltonian appear in several problems of celestial mechanics. For instance, to study the dynamics of a small particle near the equilateral libration point of the Earth-Moon system, one can take the Earth-Moon system as a restricted three-body problem (which can be written as an autonomous Hamiltonian) plus perturbations, that can be very well approximated by quasiperiodic functions, see [3].

The problem of the persistence of maximal dimensional tori of the Hamiltonian in (4.1) was first studied in [8]. Then Jorba and Villanueva [9] considered the persistence of lowerdimensional elliptic tori under the same kind of perturbations, and showed that under some hypothesis of nondegeneracy and nonresonance some of the lower-dimensional tori are not destroyed but only deformed by the perturbation with addition of the perturbing frequencies to those they had previously.

In this section, we illustrate briefly how one can directly generalize the method in section 3 to investigate the persistence of lower-dimensional hyperbolic invariant tori under time-dependent quasi-periodic perturbation.

Consider the $C^{r}$ Hamiltonian vector field with the Hamiltonian

$$
H(x, y, I, \varphi, \tilde{\varphi}, \varepsilon)=H_{0}(x, y, I)+\varepsilon H_{1}(x, y, I, \varphi, \tilde{\varphi}, \varepsilon)
$$

with respect to the symplectic form $\mathrm{d} y \wedge \mathrm{d} x+\mathrm{d} I \wedge \mathrm{d} \varphi$, where $\tilde{\varphi}$ is quasi-periodic in time with frequency $\tilde{\omega}=\left(\tilde{\omega}_{1}, \tilde{\omega}_{2}, \ldots, \tilde{\omega}_{s}\right), x, y \in R^{l}, I \in U \subset R^{k}\left(U\right.$ is an open set) and $\varphi \in T^{k}$.

For (4.2), we still suppose that (A1)-(A3) hold. In order to be able to use the method in section 3, we rewrite (4.2) as

$$
H(x, y, I, \varphi, \tilde{I}, \tilde{\varphi}, \varepsilon)=\tilde{\omega}^{\mathrm{T}} \tilde{I}+H_{0}(x, y, I)+\varepsilon H_{1}(x, y, I, \varphi, \tilde{\varphi}, \varepsilon)
$$

with the symplectic form $\mathrm{d} y \wedge \mathrm{d} x+\mathrm{d} I \wedge \mathrm{d} \varphi+\mathrm{d} \tilde{I} \wedge \mathrm{d} \tilde{\varphi}$, where $\tilde{\varphi}$ are the angle variables that denote the time, $\tilde{I}$ are the corresponding momenta and $\tilde{\omega}^{\mathrm{T}}$ denotes the transposition of $\tilde{\omega}$. Then the Hamiltonian vector field generated by $(4.3)$ is a $(2 l+2 k+2 s)$-dimensional autonomous vector field, and by the method in section 3 and the results in [8] one may obtain a conclusion similar to the one in [9].

\section{An example}

We now consider an example introduced in [7] which was used to describe an axisymmetric, translation-invariant media problem in geometrical optics.

The optical Hamiltonian with two degrees of freedom is given by

$$
H=-\sqrt{n^{2}(r)-p_{r}^{2}-p_{\varphi}^{2} / r^{2}}
$$


and Hamiltonian equations for axisymmetric translation-invariant media are expressible as

$$
\begin{aligned}
& \dot{r}=\frac{\partial H}{\partial p_{r}}=-\frac{1}{H} p_{r} \\
& \dot{p}_{r}=-\frac{\partial H}{\partial r}=-\frac{1}{2 H} \frac{\mathrm{d}}{\mathrm{d} r}\left(n^{2}(r)-\frac{p_{\varphi}^{2}}{r^{2}}\right) \\
& \dot{p}_{\varphi}=-\frac{\partial H}{\partial \varphi}=0 \\
& \dot{\varphi}=\frac{\partial H}{\partial p_{\varphi}}=-\frac{p_{\varphi}}{H r^{2}}
\end{aligned}
$$

where the physical meanings of $r \in R^{1}, p_{r} \in R^{1}, p_{\varphi} \in R^{1}, \varphi \in T^{1}$ and $n^{2}(r)$ refer to those given in [7].

Choose a radial profile of the fibre's refractive index in the following form:

$$
n^{2}(r)=\lambda^{2}+\left(\mu-v r^{2}\right)^{2}
$$

where $\lambda^{2}, \mu$ and $\nu$ are positive constants. Now we consider the following perturbations of the refractive index:

$$
n_{\varepsilon}^{2}(r)=n^{2}(r)+\varepsilon n_{0}^{2}\left(r, p_{\varphi}, \varphi\right)
$$

where

$$
n_{0}^{2}\left(r, p_{\varphi}, \varphi\right)=\left[p_{\varphi}-2 v r^{4}\left(\mu-v r^{2}\right)\right]^{9 / 2} f(\varphi)
$$

$f(\varphi)$ is a function of $\varphi$.

Expanding the Hamiltonian

$$
H_{\varepsilon}=-\sqrt{n_{\varepsilon}^{2}(r)-p_{r}^{2}-p_{\varphi}^{2} / r^{2}}
$$

in powers of $\varepsilon$, gives $H_{\varepsilon}=H_{0}+\varepsilon H_{1}$ at linear order, where

$$
H_{0}=H=-\sqrt{n^{2}(r)-p_{r}^{2}-p_{\varphi}^{2} / r^{2}} \quad H_{1}=\frac{1}{2 H_{0}} n_{0}^{2}\left(r, p_{\varphi}, \varphi\right) .
$$

This expansion is valid provided $H_{0} \gg \varepsilon$; physically, that is, away from grazing incidence to the image screen.

To linear order in $\varepsilon$, the refractive index perturbations appear in Hamiltonian equations as $\dot{r}=-\frac{1}{H} p_{r}+\varepsilon \frac{p_{r}}{2 H^{3}} n_{0}^{2}$

$\dot{p}_{r}=-\frac{1}{2 H} \frac{\mathrm{d}}{\mathrm{d} r}\left(n^{2}(r)-\frac{p_{\varphi}^{2}}{r^{2}}\right)+\varepsilon\left[\frac{1}{4 H^{3}} n_{0}^{2} \frac{\mathrm{d}}{\mathrm{d} r}\left(n^{2}(r)-\frac{p_{\varphi}^{2}}{r^{2}}\right)-\frac{1}{2 H} \frac{\partial n_{0}^{2}}{\partial r}\right]$

$\dot{p}_{\varphi}=-\frac{\varepsilon}{2 H} \frac{\partial n_{0}^{2}}{\partial \varphi}$

$\dot{\varphi}=-\frac{p_{\varphi}}{H r^{2}}+\varepsilon \frac{n_{0}^{2}}{2 H^{3}} \frac{p_{\varphi}}{r^{2}}$.

Now consider the unperturbed system of (5.8), i.e. the system (5.2). The Hamiltonian equations for $\left(r, p_{r}\right)$ in (5.8) are given by

$$
\begin{aligned}
& \dot{r}=-\frac{1}{H} p_{r} \\
& \dot{p}_{r}=-\frac{1}{H}\left[-2 v r\left(\mu-v r^{2}\right)+\frac{p_{\varphi}^{2}}{r^{3}}\right] .
\end{aligned}
$$


The equilibria of these equations occur at those points where the right-hand sides vanish, i.e. $p_{r}=0, r=\bar{r}\left(p_{\varphi}, \mu, \nu\right)$, where $\bar{r}$ satisfies the following cubic relation in $\bar{r}^{2}$ :

$$
-2 \nu \bar{r}^{2}\left(\mu-\nu \bar{r}^{2}\right)+\frac{p_{\varphi}^{2}}{\bar{r}^{2}}=0 .
$$

It is easy to check that the level surfaces of $H^{2}$ satisfy this equilibria condition when

$$
\lambda^{2}+\left(\mu-v \bar{r}^{2}\right)^{2}-H^{2}=2 v \bar{r}^{2}\left(\mu-v \bar{r}^{2}\right) .
$$

Thus if $\lambda^{2}<\frac{1}{3}\left(\mu^{2}+3 H^{2}\right)$, the equilibrium points occur in pairs, i.e.

$\bar{r}_{1}^{2}=\frac{1}{3 v}\left(2 \mu+\sqrt{\mu^{2}-3 \lambda^{2}+3 H^{2}}\right) \quad \bar{r}_{2}^{2}=\frac{1}{3 v}\left(2 \mu-\sqrt{\mu^{2}-3 \lambda^{2}+3 H^{2}}\right)$.

By the fact that a fixed point of (5.9) is stable (respectively hyperbolic), if at the point

$$
\frac{\mathrm{d}^{2}}{\mathrm{~d} r^{2}}\left(n^{2}(r)-\frac{p_{\varphi}^{2}}{r^{2}}\right)<0 \quad(\text { respectively }>0)
$$

one may find that those fixed points from pairs of roots in $\bar{r}_{1}^{2}$ are hyperbolic, while those from $\bar{r}_{2}^{2}$ are stable.

Then, we obtain a normally hyperbolic invariant manifold for the system (5.2)

$$
M=\left\{\left(r, p_{r}, p_{\varphi}, \varphi\right) \mid r^{2}=\bar{r}_{1}^{2}, p_{r}=0, p_{\varphi} \in R^{1}, \varphi \in T^{1}\right\}
$$

which consists of one-dimensional tori, i.e. periodic orbits. Our goal is to detect the persistence of these tori and their stable and unstable manifolds in the perturbed system (5.8). Due to a nonresonance condition such as (3.21), we only consider those tori with $p_{\varphi} \neq 0$.

Actually, system (5.8) is a special case of system (3.1) with $l=k=1$, and one may easily find that all the conditions required in section 3 are satisfied. Then using our method, we can show the existence of the periodic orbits and their stable and unstable manifolds for the system (5.8). However, from (5.5) and (5.10) we find that the system (5.8) is not analytical near the fixed points $r^{2}=\bar{r}_{1}^{2}, p_{r}=0$, but is $C^{3}$; namely, the previous methods are invalid for the system (5.8).

\section{Acknowledgments}

We are very grateful to the referees for their valuable comments on this manuscript. The work is supported by NNSF of China.

\section{References}

[1] Arnold V I 1963 Proof of A N Kolmegorov's theorem on the preservation of quasi-periodic motions under small perturbation of Hamiltonian Russ. Math. Surveys 18 (5) 9

[2] Cheng C Q and Sun Y S 1990 Existence of invarint tori in three dimensional measure-preserving mappings Celest. Mech. 47275

[3] Diez C, Jorba A and Simo C 1993 A dynamical equivalent to the equilateral libration points of the Earth-Moon system Celest. Mech. $\mathbf{5 0} 13$

[4] Eliasson L H 1988 Perturbations of stable invariant tori for Hamiltonian systems Ann. Scu. Norm. Super. Pisu. Cl. Sci. IV 15115

[5] Fenichel N 1971 Persistence and smoothness of invariant manifolds for flows Ind. Univ. Math. J. 21193

[6] Graff S M 1974 On the continuation of hyperbolic invariant tori for Hamiltonian systems J. Differ. Equ. 151

[7] Holm D D and Kovacic G 1991 Homoclinic chaos for ray optics in a fiber Physica D 51177 
[8] Jorba A and Simo C 1996 On quasi-periodic perturbation of elliptic equilibrium points SIAM. J. Math. Anal. 27 1704

[9] Jorba A and Villanueva J 1997 On the persistence of lower dimensional invariant tori under quasi-periodic perturbation J. Nonlinear Sci. 7427

[10] Lerman L M and Umanskii Y L 1987 Structure of the Poisson action of $R^{2}$ on a four dimensional symplectic manfold I Sel. Math. Sov. 6365

[11] Marsden J E and Ratiu T S 1994 Introduction to Mechanics and Symmetry (New York: Springer)

[12] Poschel J 1982 Integrability of Hamiltonian systems on Cantor sets Commun. Pure Appl. Math. 35653

[13] Poschel J 1989 On elliptic lower dimensional tori in Hamiltonian systems Math. Z. 213559

[14] Moser J 1966 A rapidly convergent iteration method and nonlinear differential equations, II Ann. Scu. Norm. Sup. Pisa. Ser. III 20499

[15] Rudnev M and Wiggins S 1997 KAM theory near multiplicity one resonant surfaces in perturbations of a-priori stable Hamiltonian systems J. Nonlinear Sci. 7177

[16] Spivak M 1979 Differential Geometry vol I, 2nd edn (Wilmington, IN: Publish or Perish)

[17] Wiggins S 1994 Normally Hyperbolic Invariant Manifolds in Dynamical Systems (New York: Springer)

[18] Xia Z H 1992 Existence of invariant tori in volume preserving diffeomorphisms Ergod. Theory Dynam. Syst. 12 621 